\title{
Características Morfológicas y Morfométricas de la Placenta de Término, en Recién Nacidos Pequeños para la Edad Gestacional (PEG) en la Ciudad de Temuco-Chile
}

\author{
Morphometric and Morphological Characteristics of the Placenta at Term, \\ in Small for Gestational Age Newborns (SGA) in the City of Temuco-Chile \\ "Ruth Prieto Gómez; **Fernando Matamala \& *** Mariana Rojas
}

PRIETO, G. R.; MATAMALA, F. \& ROJAS, M. Características morfológicas y morfométricas de la placenta de término, en recién nacidos pequeños para la edad gestacional (PEG) en la ciudad de Temuco-Chile. Int. J. Morphol., 26(3):615-621, 2008.

RESUMEN: El metabolismo placentario, el intercambio de sustancias y la producción de hormonas son funciones vitales de la placenta para mantener y promover el desarrollo normal del feto. Existen factores de riesgo que alteran este patrón en el caso del retardo del crecimiento intrauterino, cuyo resultado será un recién nacido $(\mathrm{RN})$ pequeño para la edad gestacional (PEG) que presentará una mayor morbilidad, crecimiento físico e intelectual comprometido y una mayor probabilidad de desarrollar durante la vida adulta diferentes patologías. Los objetivos del presente trabajo son: 1. Reconocer las diferencias en los parámetros morfométricos, como el área de las vellosidades, el área de los vasos, el número de vasos y el área del sinciciotrofoblasto de las placentas de PEG en relación con placentas de recién nacidos AEG y 2. Relacionar el diagnóstico neonatal de PEG con las características morfométricas. Se utilizaron 25 placentas de término (37-42 semanas), 12 de recién nacidos adecuados a la edad gestacional (AEG), y 13 de recién nacidos pequeños para la edad gestacional (PEG). Las muestras fueron obtenidas de la maternidad del Hospital Hernán Henríquez Aravena de Temuco, IX Región Chile. De cada placenta se tomaron dos segmentos pericordonales, desde la placa subcorial hasta la placa basal y luego fueron fijadas en formalina tamponada al 10\%. Las técnicas histológicas utilizadas fueron H-E azul de Alcián, Tricrómico de Masson, PAS-Hematoxilina y PAS-Diastasa. El área de las vellosidades mostraron diferencias significativas entre el grupo control (AEG) y el grupo PEG con $\mathrm{p}=$ 0,0194. En el grupo de PEG el área de los vasos fue significativamente mayor, con un valor de $234,05 \mu \mathrm{m}^{2}$ en comparación con el grupo control cuyo promedio fue de $150.99 \mu \mathrm{m}^{2}(\mathrm{p}=0,0001)$. El número de vasos sanguíneos por vellosidad libre no mostró diferencias significativas. En relación con el área del sinciciotrofoblasto la diferencia no resultó ser significativamente diferente $(p=0,1410)$. Como conclusión se determinó que las placentas de recién nacidos PEG presentaron diferencias significativas a nivel del área de los vasos y del área de las vellosidades coriales libres, en relación con las placentas de AEG.

PALABRAS CLAVE: Placenta; Recién nacido; Pequeño para la edad gestacional.

\section{INTRODUCCIÓN}

El peso del recién nacido es el resultado de efectos interactivos entre diferentes factores, como el potencial genético, tiempo de gestación, adecuado ambiente intrauterino, nutrición materna, salud materna y función úteroplacentaria (Kliegman \& Das, 2002).

Según Sadler (2004), los factores causales de la RCIU (restricción del crecimiento intrauterino) comprenden: anomalías cromosómicas (10\%), exposición a agentes teratogénicos, infecciones congénitas (rubéola, citomegalovirus, toxoplasmosis y sífilis), problemas de salud de la madre (hipertensión y enfermedades cardíacas y renales), el estado de nutrición de la madre y su nivel socioeconómico, el consumo de alcohol y otras drogas, la insuficiencia placentaria y embarazos múltiples (Sadler).

En muchas publicaciones científicas, los términos RCIU (restricción del crecimiento uterino) y PEG, se uti-

\footnotetext{
* Depto. de Pediatría, Universidad de La Frontera.

** Depto. de Cs. Básicas, Fac. de Medicina, Universidad de La Frontera.

**** Programa de Anatomía y Biología del Desarrollo, Fac de Medicina, Universidad de Chile.

Patrocinio: Proyecto de investigación DIUFRO 120633.
} 
lizan indistintamente para describir el mismo fenómeno, sin embargo, estos dos términos no son sinónimos. Se define como "restricción del crecimiento intrauterino", una reducción del patrón de crecimiento fetal esperado, y es el resultado de procesos que inhiben el crecimiento potencial del feto (Kliegman \& Das). El término "pequeño para la edad gestacional" (PEG) se define como un niño cuyo peso al nacimiento esta bajo el percentil 10 para su edad gestacional, y el término "bajo peso al nacer" se define como el peso al nacer menor que $2 \mathrm{Kg}$ (Frisbie et al., 1996; Kliegman \& Das). Existen patologías como la diabetes, la lúes y la enfermedad hemolítica severa por factor Rh que se asocian a placenta de mayor tamaño; mientras que el síndrome hipertensivo del embarazo, el retardo del crecimiento intrauterino y la inmadurez se asocian a placenta de menor peso y tamaño (Las Heras et al., 1999).

En la gestación avanzada, los capilares fetales de la barrera placentaria (capilares continuos) se observan dilatados, los pericitos desaparecen de las zonas alfa (láminas sinciciovasculares) y las membranas basales tanto de estos capilares, como del trofoblasto, se engruesan por depósitos de colágeno IV. A su vez, aumenta el depósito de material fibrinoide en las vellosidades coriónicas y de colágeno tipo I en el tejido conjuntivo de las estructuras. Todo lo anterior se debe al envejecimiento del órgano. Esto tiene importancia en el diagnóstico clínico, pues permite informar el grado de maduración del órgano a través de la biopsia de placenta (Bosco, 2003).

La arquitectura vascular dentro de la vellosidad ha sido analizada por cuantificación estereológica en cortes de tejidos placentarios por Charnock-Jones et al. (2004) y Kauffmann et al. (2004) En nuestro estudio realizaremos una cuantificación de parámetros morfológicos, relacionados con área de las vellosidades y áreas de vasos sanguíneos de PEG en comparación con las placentas de AEG.

En la literatura revisada de los últimos 10 años, no se ha encontrado estudios detallados que muestren los cambios morfológicos, en cada uno de los compartimentos placentarios en los casos de recién nacidos PEG. Tampoco hay un acuerdo en la literatura sobre el significado microscópico de la insuficiencia placentaria. Actualmente se atribuye este diagnóstico a la RCIU cuando se descartan causas maternas u ovulares, según normas.

De esta manera, el propósito del estudio fue reconocer las diferencias en los parámetros morfométricos de las placentas de PEG en relación con placentas control, como también, relacionar el diagnóstico neonatal de PEG con las características morfométricas de la placenta.

\section{MATERIAL Y MÉTODO}

Se utilizaron 25 placentas de término (37-42 semanas), provenientes de gestaciones únicas y sin malformaciones, 12 de ellas de AEG y 13 de recién PEG, obtenidas del Hospital Hernán Henríquez Aravena de Temuco, IX Región Chile. Se utilizó una ficha de registro clínico para consignar: peso del primer y último control, talla materna, edad, etnia (mapuche o no mapuche), número ordinal del parto, patologías asociadas a la gestación. Del recién nacido se consignó sexo, peso y talla. Los recién nacidos bajo el percentil 10 fueron clasificados como PEG.

De las placentas se midió el diámetro y peso, luego fueron fijadas en formalina tamponada al $10 \%$; obteniéndose 20 placas histológicas de cada muestra. Se utilizaron técnicas histológicas como H-E azul de Alcián, Tricrómico de Masson PAS-Hematoxilina y PAS-Diastasa.

A continuación, se analizaron las secciones histológicas de cada muestra de placenta AEG y de placenta PEG, utilizando un Microscopio NIKON y una cámara digital incluida NIKON E 4.500. Se seleccionaron las vellosidades libres cortadas en forma transversal y se consideraron los siguientes parámetros para ser evaluados: a) área de la vellosidad b) Número de vasos sanguíneos en cada vellosidad. c) Diámetro de los vasos sanguíneos de la vellosidad (para calcular el área de la vellosidad ocupada por vasos sanguíneos). Para la morfometría se utilizó el programa computacional Image Tool. Para determinar si las diferencias encontradas entre las placentas normales y PGE tuvieron significancia estadística, se utilizaron las pruebas de t de Student y Programa GraperPad Prisma 4. Los resultados se presentan como SEM (Error Estándar Medio, que es la diferencia que en promedio hay en cada dato aislado y la media), promedio, y aquellos valores de $\mathrm{p} \leq 0.05$, se consideraron estadísticamente significativos.

\section{RESULTADOS}

El peso promedio de recién nacidos del grupo control fue de $3045 \mathrm{~g}$ con un rango entre $2710 \mathrm{~g}$ y $3900 \mathrm{~g}$. El peso promedio de la placenta fue de $495 \mathrm{~g}$ con un rango entre 400 y $720 \mathrm{~g}$. El peso promedio de RN PEG fue $2448 \mathrm{~g}$ con un rango entre 1555 y $2940 \mathrm{~g}$. El peso promedio de las placentas en los PEG fue de $386.15 \mathrm{~g}$ con un rango entre 280 y $570 \mathrm{~g}$.

Las características macroscópicas eran normales, con inserción excéntrica del cordón umbilical, sólo en dos casos 
se observó placentas en raqueta, un caso de lobulación de la placenta y uno de inserción velamentosa. El diámetro promedio entre ambos bordes de las placentas AEG fue de $19,14 \mathrm{~cm}$, con un rango entre 17 y $22 \mathrm{~cm}$; en las de placentas PEG el diámetro promedio fue de $15,73 \mathrm{~cm}$, con un rango de 14 y $21 \mathrm{~cm}$.

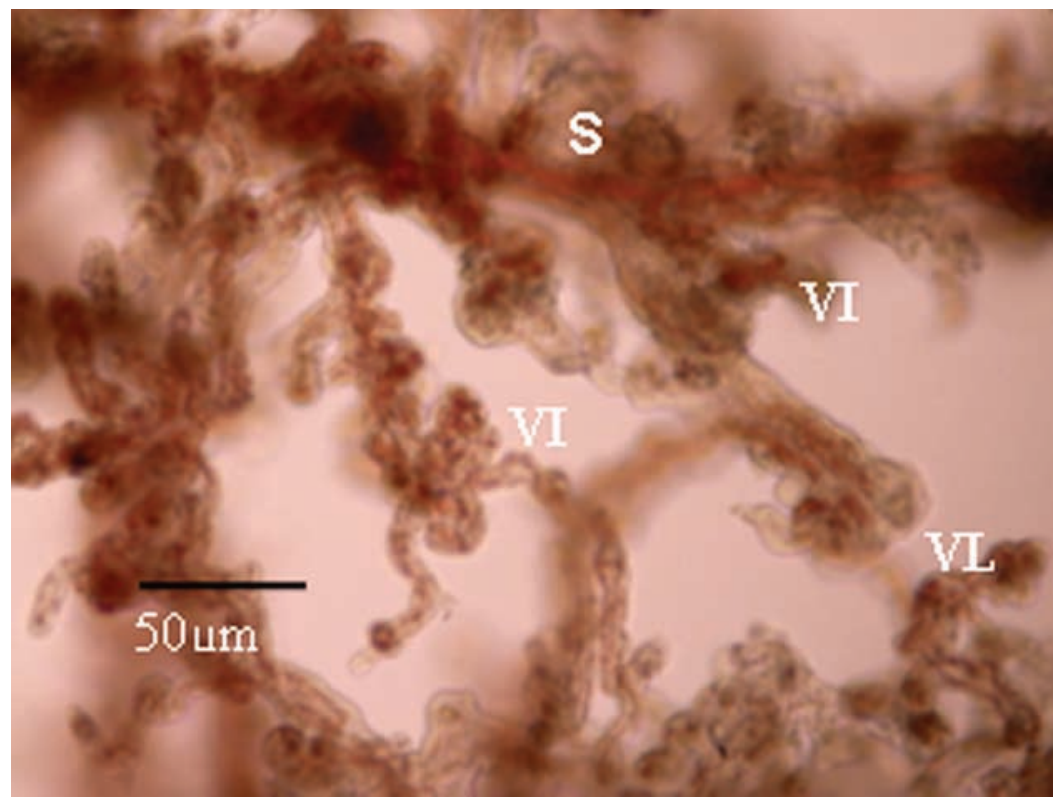

Fig. 1. Vellosidades coriales de placenta fresca "in toto". Se observa VI: vellosidades intermedias, V.L: vellosidades libres. S: capilar sanguíneo. 100X.
Las Tablas I y II muestran el peso de la placenta y peso del recién nacido vs peso materno durante el embarazo en los recién nacidos PEG y AEG, respectivamente.

En cuanto al área de las vellosidades coriónicas, estas se pueden verificar en la tabla III, donde se aprecia que la superficie libre de la vellosidad depende del área que ocupan los vasos y del área del sinciciotrofoblasto. Además, un tercio del área de la vellosidad corresponde en promedio al área del sinciciotrofoblasto. Entendemos por área libre de la vellosidad, el tejido donde el sinciciotrofoblasto y los vasos sanguíneos están ausentes.

El área de las vellosidades muestra diferencias significativos entre los grupos control $(1611+645)$ y PEG $(2547+1066) \mathrm{p}=$ 0,0194. En el grupo de PEG el área ocupada por los vasos resultó ser significativamente mayor, $\mathrm{p}=0,0001$, con un valor de 234,05 $\mu \mathrm{m}^{2}$ en comparación con del grupo control que fue $150,99 \mu \mathrm{m}^{2}$. El número de vasos sanguíneos por vellosidad libre no mostró diferencias significativas obteniéndose en el grupo control un promedio de 4 vasos de distinto calibre, en comparación con 5-6 obteni-

Tabla I. Peso de la placenta y peso del recién nacido vs peso materno durante el embarazo en recién nacidos PEG.

\begin{tabular}{lcccccccccccc}
\hline $\mathbf{N}^{\mathbf{m}}$ muestra & $\mathbf{1}$ & $\mathbf{2}$ & $\mathbf{3}$ & $\mathbf{4}$ & $\mathbf{5}$ & $\mathbf{6}$ & $\mathbf{7}$ & $\mathbf{8}$ & $\mathbf{9}$ & $\mathbf{1 0}$ & $\mathbf{1 1}$ & $\mathbf{1 2}$ \\
\hline *Peso materno & $\mathrm{D} / \mathrm{D}$ & $\mathrm{B} / \mathrm{B}$ & $\mathrm{B} / \mathrm{B}$ & $\mathrm{B} / \mathrm{B}$ & $\mathrm{B} / \mathrm{B}$ & $\mathrm{B} / \mathrm{C}$ & $\mathrm{A} / \mathrm{A}$ & $\mathrm{C} / \mathrm{C}$ & $\mathrm{B} / \mathrm{C}$ & $\mathrm{C} / \mathrm{C}$ & $\mathrm{C} / \mathrm{C}$ & $\mathrm{A} / \mathrm{B}$ \\
Inicio/término embarazo & 2650 & 2940 & 2700 & 2240 & 2410 & 2535 & 2500 & 2200 & 2565 & 2610 & 2200 & 2730 \\
Peso del RN (kg) & 440 & 430 & 400 & 360 & 340 & 460 & 390 & 350 & 340 & 340 & 280 & 470 \\
Peso placenta (g) & $1 / 6$ & $1 / 7$ & $1 / 7$ & $1 / 6$ & $1 / 7$ & $1 / 5$ & $1 / 6$ & $1 / 6$ & $1 / 7$ & $1 / 8$ & $1 / 8$ & $1 / 6$ \\
Relación: Peso RN/peso placenta & $\mathrm{X}$ & & & $\mathrm{X}$ & $\mathrm{X}$ & & & $\mathrm{X}$ & $\mathrm{X}$ & & $\mathrm{X}$ & \\
RCIU (diagnóstico prenatal) & $\mathrm{X}$ & & & & & & & & & &
\end{tabular}

*Peso materno de acuerdo a evaluación nutricional durante el embarazo: A = Bajo peso; $\mathrm{B}=$ Peso Normal; C = Sobrepeso; D = Obesa. RCIU: Restricción del Crecimiento Intrauterino. PEG: Pequeño para la edad gestacional.

Tabla II. Peso de la placenta y peso del recién nacido vs peso materno durante el embarazo en recién nacidos AEG.

\begin{tabular}{lllllllllllll}
\hline $\mathbf{N}^{\mathbf{0}}$ muestra & $\mathbf{1}$ & $\mathbf{2}$ & $\mathbf{3}$ & $\mathbf{4}$ & $\mathbf{5}$ & $\mathbf{6}$ & $\mathbf{7}$ & $\mathbf{8}$ & $\mathbf{9}$ & $\mathbf{1 0}$ & $\mathbf{1 1}$ & $\mathbf{1 2}$ \\
\hline $\begin{array}{l}\text { Peso materno } \\
\text { Inicio/término embarazo }\end{array}$ & $\mathrm{A} / \mathrm{A}$ & $\mathrm{D} / \mathrm{D}$ & $\mathrm{A} / \mathrm{B}$ & $\mathrm{A} / \mathrm{C}$ & $\mathrm{B} / \mathrm{B}$ & $\mathrm{D} / \mathrm{C}$ & $\mathrm{B} / \mathrm{C}$ & $\mathrm{B} / \mathrm{B}$ & $\mathrm{B} / \mathrm{A}$ & $\mathrm{C} / \mathrm{C}$ & $\mathrm{D} / \mathrm{D}$ & $\mathrm{D} / \mathrm{D}$ \\
Peso del RN (kg) & 3050 & 3400 & 3350 & 3000 & 3800 & 3380 & 3520 & 3450 & 3660 & 3900 & 3230 & 2710 \\
Peso placenta (g) & 490 & 440 & 470 & 490 & 530 & 500 & 530 & 500 & 460 & 720 & 470 & 400 \\
Relación: Peso RN/peso placenta & $1 / 6$ & $1 / 8$ & $1 / 7$ & $1 / 6$ & $1 / 7$ & $1 / 7$ & $1 / 7$ & $1 / 7$ & $1 / 8$ & $1 / 5$ & $1 / 7$ & $1 / 7$ \\
\hline
\end{tabular}

Peso materno de acuerdo a evaluación nutricional durante el embarazo: $\mathrm{A}=$ Bajo peso; $\mathrm{B}=$ Peso Normal; $\mathrm{C}=$ Sobrepeso; $\mathrm{D}=\mathrm{Obesa}$. RN: Recién nacido AEG: Adecuado para la edad gestacional. 
PRIETO, G. R.; MATAMALA, F. \& ROJAS, M. Características morfológicas y morfométricas de la placenta de término, en recién nacidos pequeños para la edad gestacional (PEG) en la ciudad de Temuco-Chile. Int. J. Morphol., 26(3):615-621, 2008.

Tabla III. Comparación entre el área de las vellosidades coriónicas, el área del sinciciotrofoblasto, el número de vasos por vellosidad y el área de los vasos entre placentas AEG y placentas de PEG.

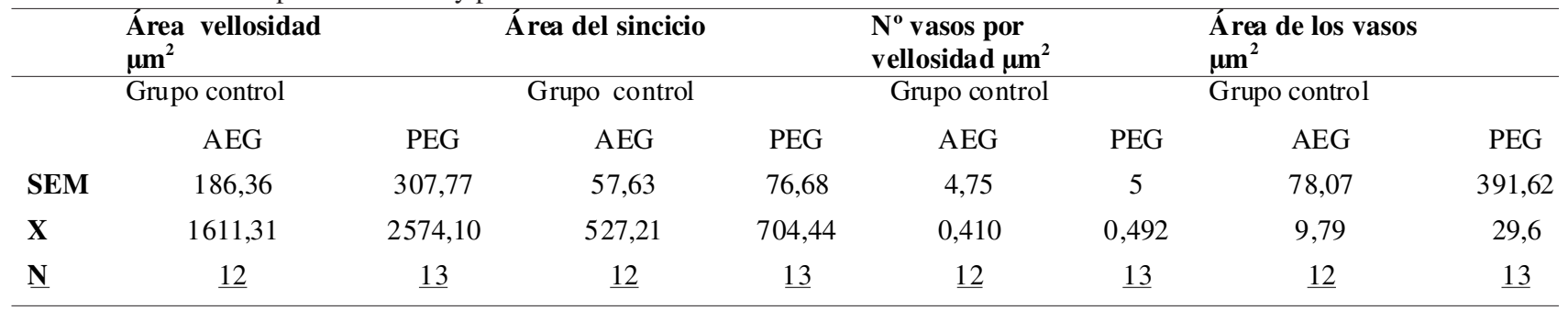

$\mathrm{SEM}=$ Error estándar medio, diferencia que en promedio hay en cada dato aislado y la media (x).

dos de los PEG. En relación con el área del sinciciotrofoblasto la diferencia no resultó ser significativa, $\mathrm{p}=0,0736$, y los valores fueron de $527+199 \mu \mathrm{m}^{2}$ en el grupo control vs 707+265 en el grupo PEG.

Morfología de las vellosidades de placentas PEG: El estroma de la vellosidad tiene aspecto más indiferen- ciado, (con escasa cantidad de células y fibras colágenas) que los controles. La matriz extracelular es rica en glicógeno y glicoproteinas evidenciables con la técnica PAS y PAS Diastasa. En el estroma se aprecian una a tres células de citotrofoblasto. El fibrinoide se observa con técnica corriente y PAS en las vellosidades troncales. Todas las técnicas utilizadas permiten visualizar las zonas
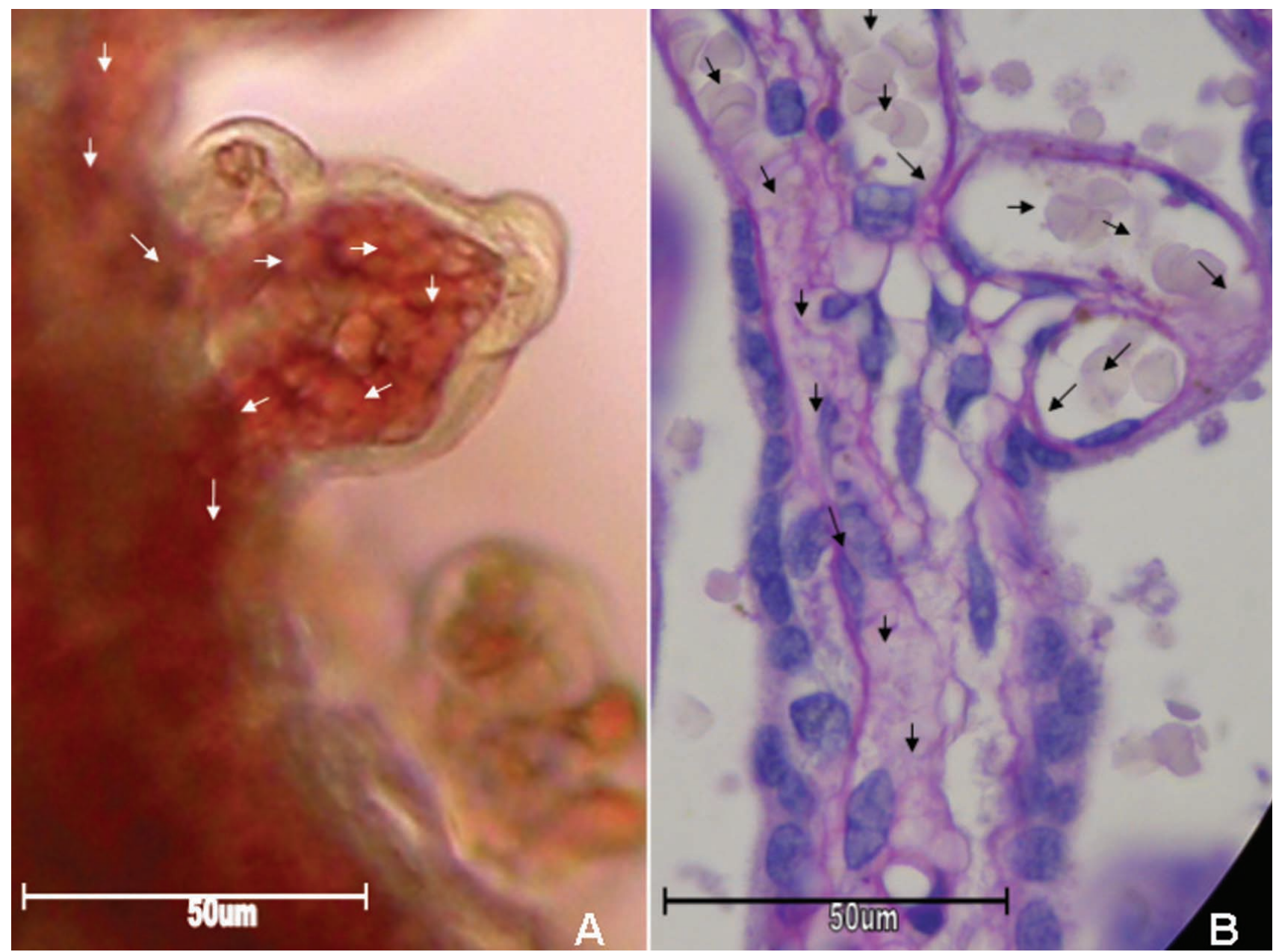

Fig. 2. A. Trayecto del capilar sanguíneo en la vellosidad terminal (flechas). B. Trayecto de vasos sanguíneos en las vellosidades (flechas) corte histológico H-E. 1000X. La barra corresponde a $50 \mu \mathrm{m}$. 

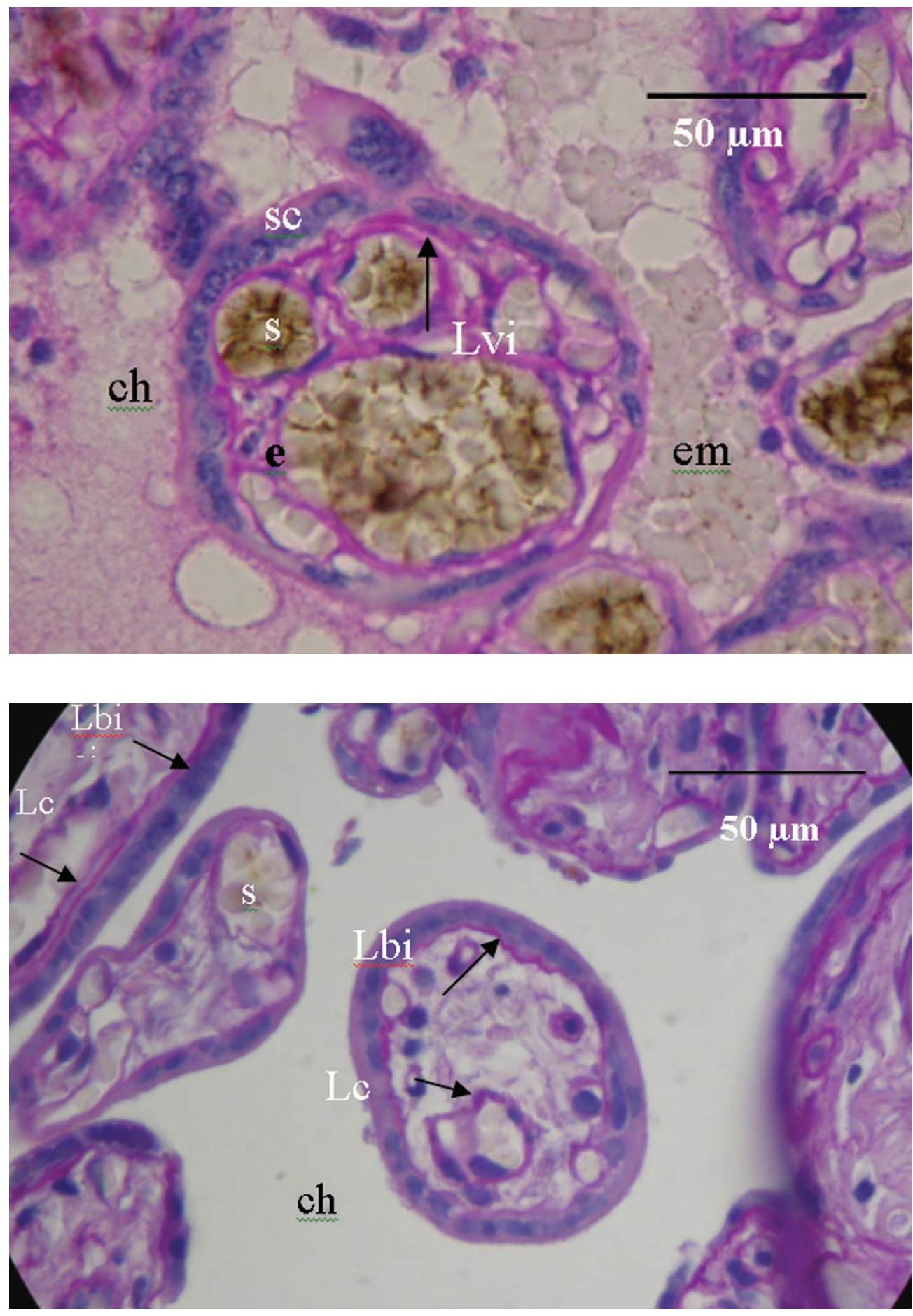

Fig. 3. Vellosidades coriales libres de placenta de término de PEG. ch: cámara hemática, em: eritrocitos maternos, sc: sinciciotrofoblasto, s: capilar sanguíneo, e: estroma. Lbi: indica la lámina basal de la interfase entre trofoblasto y estroma.

La barra corresponde a $50 \mu \mathrm{m}$. PAS. (1000X).
Fig. 4. Vellosidades coriales de placentas de término AEG. ch: cámara hemática, e: estroma constituido por fibras colágenas, Lc: lámina basal del capilar. Lbi: lámina basal de la interfase trofoblastoestroma, S: capilar sanguíneo. A) H- E, azul de Alcián. (1000X).

La barra corresponde a $50 \mu \mathrm{m}$. alfa y beta de la vellosidad, como también la lámina sincicio vascular. En la cámara hemática se identifican eritrocitos. Una lámina basal fácilmente identificable limita al sincicio y la pared de los vasos sanguíneos. Los vasos sanguíneos se observan de distintos calibres en número de seis a ocho (Fig. 3).
Morfología de las vellosidades de placenta AEG: Se observan entre cuatro a seis vasos sanguíneos por vellosidad, las membranas basales son más delgadas que en el grupo PEG, y el estroma es más denso, con técnica Tricrómico de Masson se evidencia una mayor cantidad de fibras colágenas que se disponen densamente (Fig. 4). 


\section{DISCUSIÓN}

Las placentas estudiadas mostraron que el peso y diámetro promedio de las placentas del grupo PEG resultaron ser menores que las del grupo AEG. Por otra parte el análisis de nuestros resultados demuestra que las vellosidades coriales libres, que provienen de placentas PEG, presentan diferencias morfométricas significativas en dos de los cuatro parámetros evaluados: mayor área de las vellosidades terminales y mayor área de los vasos sanguíneos de estas mismas vellosidades, en relación con las placentas normales. El número de vasos sanguíneos por vellosidad no presentó diferencias significativas pero sí se observó una mayor tendencia de estos en los PEG. Por otra parte, Castejon et al. (2005) han observado que los vasos vellositarios en embarazadas hipertensas pierden su tensión mecánica, el diámetro de los capilares disminuye cada vez más hacia las vellosidades terminales, se torna irregular y los capilares disminuyen en número. El diámetro capilar en las vellosidades terminales por ejemplo, se reduce de $24.8 \mu^{2}$ en placentas de pacientes normales hasta $13,8 \mu \mathrm{m}^{2}$ en placentas asociadas a muerte fetal intrauterina, hipertensión inducida por el embarazo donde se reduce el número de vellosidades intermedias. El daño endotelial y demás cambios patológicos son producto de una reacción inflamatoria generalizada con participación del sistema de coagulación (Castejon).

Según Torry et al. (2004) Los capilares fetales de la placenta humana no son estructuras estáticas y pueden adaptarse a una variedad de cambios y stress. Estos autores han asociado a los cambios en el desarrollo vascular de la placenta, la RCIU, los abortos espontáneos y la preeclamsia. Chen et al. (2002) realizaron comparaciones inmunohistoquí- micas de placenta de tercer trimestre con RCIU severo o normal y observaron una disminución significativa en el número de capilares vellositarios por unidad de estroma y también una disminución en el área del capilar en relación con el estroma placentario. No se conoce cuáles son los mecanismos responsables de la falta de formación de vasos en el RCIU/PEG, pero al parecer sería un defecto en la producción del más potente factor de crecimiento angiogénico VEGF (IGF-I Ó II) (Lash et al., 2001). Los capilares periféricos en la vellosidad terminal no están representados por una red ricamente ramificada sino que esta compuesto por un gran número de capilares elongados y moderadamente ramificados.

Fue posible observar en esta investigación, una reducción de las láminas basales de las vellosidades coriales de los PEG en comparación con las vellosidades coriales de controles. Según Bernirschke \& Kaufman (1999) la lámina basal trofoblástica separa el epitelio trofoblástico del estroma vellositario y está constituido por colágeno IV, laminina, heparán sulfato, y puede asociarse con otras proteínas de la matriz extracelular como fibronectina y tenascina. Se conoce también que la lamina basal trofoblástica presenta proteoglucanos polianiónicos que son responsables de impedir, como una barrera, la entrada de macromoléculas cargadas negativamente hacia el estroma (King, 1985).

Como conclusión, se determinó que las placentas de recién nacidos PEG presentaron diferencias significativas a nivel del área de los vasos y del área de las vellosidades coriales libres, en relación con las placentas de AEG.

PRIETO, G. R.; MATAMALA, F. \& ROJAS, M. Morphometric and morphological characteristics of the placenta at term, in small for gestational age newborns (SGA) in the city of Temuco-Chile. Int. J. Morphol., 26(3):615-621, 2008.

SUMMARY: The placental metabolism, the exchange of substances and the production of hormones are vital functions of the placenta to maintain and promote the normal development of the fetus. There are risk factors that disrupt this pattern in the case of intrauterine growth retardation, whose outcome will be a small for gestational age (SGA) newborn having a higher morbidity, physical and intellectual growth pledged and greater probability of develop different pathologies during adulthood. The aims of this study are:1.recognize the morphometric parameters differences as the area of the villi, the area of the vessel, the number of vessels and the area of placental syncytiotrophoblast SGA in connection with placentas of newborns AGA and 2.- relate the diagnosis of neonatal SGA with morphometric characteristics. We used 25 placenta at term (37-42 weeks), 12 newborns appropriate to the gestational age (AEG), and 13 small for gestational age infants (SGA). The samples were obtained from the Maternity Hospital Hernan Henriquez Aravena of Temuco, Chile IX Region. In each placenta two segments were taken from the subchorionic plate to the basal plate and then were fixed in $10 \%$ formalin buffered. The histological techniques used were H- E Alcián blue, Masson's Trichromic, Pas-hematoxylin Pas-diastase. The area of the villi showed significant differences between the control group (AEG) and the PEG group with $\mathrm{p}=0.0194$. In the group of PEG the area of vessels was significantly higher, with a value of $234.05 \mathrm{~mm}^{2}$ compared with the control group whose average was $150.99 \mathrm{~mm}(\mathrm{p}$ $=0.0001)$. The number of blood vessels for free villi sampling not significant differences. Regarding the area of syncytiotrophoblast the difference was not significantly $(\mathrm{p}=0.1410)$. In conclusion it was determined that PEG newborns placenta showed significant differences at the blood vessel area and free chorial villi area in relation to the AEG placenta.

KEY WORDS: Placenta; Newborn; Small for gestational age. 


\section{REFERENCIAS BIBLIOGRAFICAS}

Bernirschke, K. \& Kaufman, P. Pathology of the human placenta. $4^{\text {th }}$ Ed. New York, Springer-Verlag, 1999. pp.523-90.

Bosco, C. La placenta. En: Embriología Humana. Ediciones Universidad de Chile, Santiago, 2003.

Castejon, O. C. La lámina basal trofoblástica y su relación con la ultraestructura del trofoblasto. Gac. Méd. Caracas 113(1):65-7, 2005.

Chen, C.P.; Bajori, R. \& Aplin, J. D. Decreased vascularization and cell proliferation in placentas of intrauterine growth-restricted fetuses with abnormal umbilical artery flow velocity waveforms. Am. J. Obstet. Gynecol., 187(3):764-9, 2002.

Chernock-Jones, D. S.; Kaufmann, P. \& Mayhew, T. M. Aspects of human fetoplacental vasculogenesis and angiogenesis. I Molecular regulation. Placenta, 25(23):103-13, 2004.

Frisbie, W. P.; Forbes, D. \& Pullum, S. G. Compromised birth outcomes and infant mortality among racial and ethnic groups. Demography, 33(4):469-81, 1996.

Kaufmann, P.; Mayhew, T. M. \& Chernock-Jones, D. S. Aspects of human fetoplacental vasculogenesis and angiogenesis. II changes during normal pregnancy. Placenta 25(2-3):114-26, 2004.

King, B. F. Distribution and characterization of sites in trophoblast and capillary basal laminas of human placental villi. Anat. Rec., 212(1):63-8, 1985.

Kliegman, R. M. \& Das, U. G. Intrauterine growth retardation. In: A. A. Fanaroff \& R. J. Martin (Eds.), Neonatal-perinatal medicine: Diseases of the newborn. St. Louis, Mosby, 2002. pp.228-62.

Las Heras, J.; Dabancens, A. \& Seron Ferre, M. Placenta y anexos embrionarios. En:Pérez-Sánchez, A. \& Donoso Siña. E. Obstetricia. $3^{\text {a }}$ ed. Santiago, Editorial Mediterráneo, 1999.

Lash, G.; Macpherson, A.; Liu, D.; Charnock-Jones, S. \& Beker, P. Abnormal fetal growth is not associated with altered chorionic villous expression of vascular endothelial growth factor mRNA. Mol. Hum. Reprod., 7(11):1093-8, 2001.
Sadler, T. W. Langman Embriología médica con orientación clínica. $9^{a}$ Ed. Madrid, Editorial Médica Panamericana, 2004.

Torry, D. S.; Hinrichs, M. \& Torry, R. J. Determinants of Placental Vascularity. Am. J. Reprod. Immunol., 51(4):257-68, 2004.

\author{
Dirección para correspondencia: \\ Ruth Prieto Gómez \\ Departamento de Pediatría y Cirugía Infantil \\ Facultad de Medicina \\ Universidad de La Frontera \\ Manuel Montt 115 \\ Temuco \\ CHILE \\ Email: rprieto@ufro.cl
}

Recibido : 03-01-2008

Aceptado: 01-07-2008 
